\title{
Lola Pons Rodríguez \\ En torno al marcador de topicalización respecto: variantes y uso en sincronía
}

https://doi.org/10.1515/zrp-2018-0069

\begin{abstract}
This paper provides a description of the use in Spanish of the discourse particle respecto as a topicalizer. Although it has undergone complete grammaticalization from Latin in Spanish it appears in many forms. The distribution of the different forms involved is analysed using synchronic data from the CREA corpus and other lexicographical evidence.
\end{abstract}

Keywords: topicalization, topicalizer, aboutness, discourse particles, Spanish language

Palabras clave: topicalización, topicalizador, respectividad, partículas discursivas, lengua española

\section{Topicalización en el español de hoy y de ayer}

Un siglo después de una aproximación pretéorica pero de notable lucidez, como la de la obra De l'ordre des mots dans les langues anciennes comparées aux langues modernes de Henri Weil (1844), los trabajos realizados por Mathesius y la continuidad que estos encuentran en la Escuela de Praga suponen el arranque de una preocupación por la estructura informativa que ha dado continuos frutos hasta la actualidad. En este ámbito, el interés de los estudiosos se ha concentrado de manera bastante uniforme en unos mismos fenómenos, entre los que sobresalen los siguientes: la posición relativa de los elementos dentro de la frase, con atención especial a las posiciones desgajadas, a la izquierda o a la derecha; los fenómenos de recuperación pronominal de las secuencias desgajadas (reduplicaciones, doblado de clíticos); las propiedades de tales elementos destacados en la frase (referencialidad, definitud...) y, desde una perspectiva más amplia que la de la organización interna de los enunciados, el análisis del encadenamiento informativo de los textos (por ejemplo a través de estudios sobre progresión temática).

Dirección de correspondencia: Prof. Dr. Lola Pons Rodríguez, Universidad de Sevilla, Departamento de Lengua Española, Lingüística y Teoría de la Literatura, C/ Palos de la Frontera s/n, E-41004 Sevilla, E-Mail: lolapons@us.es 
Recientes aportaciones al tema como las de Ferrari/Borreguero Zuloaga (2008) o los estudios recopilados por Dufter/Octavio de Toledo (2014) han puesto de nuevo en el primer plano la discusión sobre la estructura informativa del texto y la relevancia que la posición izquierda tiene como marco y periferia caracterizadora de la estructura tipológica de una lengua como el español.

\subsection{Qué entendemos por topicalizar}

La existencia de un ámbito de preocupaciones más o menos amplio, pero también más o menos común, no ha contribuido, sin embargo, a la uniformidad terminológica de los trabajos. Las grandes corrientes de estudios sobre la estructura informativa (praguenses: Mathesius, Daneš, Firbas, Svoboda; funcionalistas: Halliday, Dik, Givón o generativistas como Dahl ${ }^{1}$ ) se sirven de una serie de pares conceptuales diversos: tema / rema, tópico / comentario, información nueva / dada, foco neutro / contrastivo... en un bosque de denominaciones bastante complejo al que habrá que sumar, para el caso de la investigación hecha en español, el problema venido de la propia diversidad en el volcado a nuestro idioma de términos de otras lenguas (inglés, francés, pero también checo en el caso de los trabajos de Mathesius).

En este trabajo entendemos tópico como un miembro oracional con capacidad metadiscursiva, que delimita el ámbito sobre el que va a tratar el enunciado siguiente:

(1) En cuanto al curso actual, Luis mejoró su puesto, pero Pepa no.

en (1), en cuanto al curso actual posee la propiedad de la respectividad (traducción del inglés aboutness) y delimita un marco discursivo sobre el que trata lo que sigue; el enunciado Luis mejoró su puesto, pero Pepa no es pertinente en relación con dicho marco, pero no lo sería con un marco discursivo diferente. La tradición anglosajona generó el término topic para aludir a este marco, que se ha confundido en muchas ocasiones con el de tema (desde theme, denominación venida del círculo praguense), aun siendo conceptos distintos. ${ }^{2}$ Ante la diversidad de deno-

1 Una aproximación historiográfica muy útil puede verse en los capítulos introductorios de Hidalgo Downing (2003) y en varios de los trabajos de Borreguero Zuloaga (entre ellos, 2004).

2 En español, para aumentar más el ya de por sí enmarañado muestrario terminológico, tema se ha empleado como traducción tanto de theme como del propio topic («porque resulta una traducción más natural» Hidalgo Downing 2003, 42 n. 1). La dificultad se acrecienta si consideramos dos hechos sobre los que llama la atención Borreguero Zuloaga (2004, 448 n. 4): que Daneš 
minaciones que pueden hallarse en los trabajos sobre estructura informativa, en este trabajo se adoptan las siguientes decisiones terminológicas:

(i) Siguiendo a Halliday (1967), postulo la separación de tema / rema (en el sentido praguense o en el que aquí le daremos) frente a información dada (¿o nueva?) / información conocida, que funcionan en un nivel textual y que se articulan de forma independiente a la separación entre nuevo / dado. Por ello, no asimilamos rema a información nueva ni tema a información conocida.

(ii) Concibo como nociones distintas tema y tópico, pero siguiendo a Hidalgo Downing $(2003,42)$ apuesto por tópico y topicalización por razones de extensión de uso, aunque algunos autores prefieran hablar de tematización por norma lingüística. ${ }^{3}$ La topicalización como procedimiento implica la proyección sintáctica de la estructuración informativa del predicado, y esa proyección puede quedar satisfecha de manera inequívoca con el uso de una expresión como la que se analiza en este trabajo, respecto. Debemos subrayar que en la escasa bibliografía que específicamente se dedica a las propiedades de la topicalización con expresiones ad hoc no queda claro si estas presentan distintas propiedades discursivas que la topicalización sin índices introductorios claramente metadiscursivos. Quienes le atribuyen propiedades específicas señalan una posibilidad de recuperar un tema olvidado circunstancialmente en el discurso, pero las opiniones no son coincidentes entre autores:

equipara tema con tópico y rema con comentario y el que, a la inversa, Dik llama theme al tópico y topic al tema. El tópico puede coincidir en algunos autores con el tema, entendido, según la propuesta praguense y desde la adaptación que hizo Firbas (1964) de las denominaciones de Mathesius como la entidad que centra la estructura informativa de la frase y de la que el rema practica una aseveración de modalidad discursiva variable. En El curso actual, la gente lo ve con esperanza y yo con abatimiento, el tema es el curso actual y puede entenderse que el tópico (aquello de lo que trata la frase) es también el curso actual. En (1), en cambio, al tópico el curso actual le siguen el tema Luis y el rema Pepa. Además, hay que tener presente la existencia de focos, que de forma no obligatoria, pueden superponerse a las estructuras temático-remáticas, para señalar «la parte de la oración que no está presupuesta en un contexto determinado o que presenta la información de una nueva manera» (Beaudrie 2005, 1). Las estrategias de focalización dotan de relieve a un constituyente de la oración por centrarse en él la información, y esa saliencia puede asignarse contextualmente, mediante la entonación o con indicios sintácticos como la aparición de estructuras ecuacionales (A la que vi ayer en casa fue a Blanca) o propiamente de adverbios de focalización.

3 «Utilizamos el término tema porque pensamos que se trata de una traducción más natural al español y porque este término se emparenta mejor con la dimensión discursiva del tema en su uso corriente, como 'aquello de lo que hablamos' y que se manifiesta en la organización temática de la interacción hablada» (Hidalgo Downing 2003, 46). 
«[E]n nuestros datos la tematización introducida por estas expresiones no reintroduce asuntos relegados sino que prepara, en forma de marco, la introducción de un asunto nuevo en el discurso. Estos marcos tienen como propósito proporcionar al oyente ciertas indicaciones metatextuales acerca de la organización del habla; en este caso, el hablante anuncia su intención de iniciar un nuevo tema» (Hidalgo Downing 2003, 186s.).

(iii) Nos acercaremos a los topicalizadores como elementos al servicio de la respectividad; el valor praguense de tema, asociado a los elementos iniciales de cláusula, queda disociado de esa noción de respectividad (aboutness) pero tal respectividad no asume en sí forzosamente al tema. Esto es, en un frase como (1) la cláusula con en cuanto a introduce una referencia metadiscursiva seguida de un tema y un rema.

Partiendo de la idea de tópico trazada, separamos varios procedimientos de creación de tema o tematización. El más inmediato es el de la entonación, como la marcación prosódica desgajada: Martín Butragueño $(2006,3)$ hablaba de «encorchetamiento prosódico» de la cláusula tematizada, separación que se singularizaba de forma diversa (ib., 56). Otra posibilidad es el cambio en el orden de palabras: pueden darse tópicos integrados, sin dislocación posicional (Tú a Boston y yo a California). En otros casos, sí es posible hablar de cambio en el orden de palabras, por ejemplo hacia la anteposición, que, además, puede implicar un elemento anafórico. Si es así, se habla de esta tematización como dislocación a la izquierda (sin reasunción clítica, se ha hablado a veces de topificación). ${ }^{4}$ La frontalización que sufre el elemento o sintagma topicalizado no lo convierte, pues, en independiente sintácticamente, ya que pueden darse vinculaciones sintácticas a través de copias pronominales, reaparición en forma de demostrativos o incluso mediante una unidad léxica coincidente o no con lo tematizado:

(2) a. La tesis, Raquel la defendió solventemente.

b. Y de la polémica del otro día, qué quieres que te diga, yo paso de esa historia.

c. Lo que es a festejos, Pepa ha llevado a Luis a muchas ferias.

d. Con respecto a los alumnos, ahora empiezan a hacer sus prácticas.

El alcance extraproposicional del sintagma topicalizado suele plasmarse, como se observa en los ejemplos anteriores, en una colocación a la izquierda de la predicación. Debe darse una condición de pertinencia entre lo tematizado y la

4 La discusión sobre las funciones de esas dislocaciones a la izquierda ha sido mucha. Se apunta a factores expresivos como el énfasis y el contraste (Silva-Corvalán 1984; Fernández Soriano 1993; Mendieta/Molina 1997) o, como hace Hidalgo Downing (2003) al establecimiento inequívoco del tema del enunciado. 
cláusula que sigue, que aportará información relevante sobre la entidad destacada. Ello explica, por un principio de iconicidad cognitiva, que se sitúe delante del predicado que aporta información. El sintagma tematizado señalará al ámbito afectado por esa predicación o el marco en el que esa predicación resulta de interés. Se ha propuesto la existencia de topicalización a la derecha (3a) en la que parece difícil la viabilidad de inserción de una expresión específicamente tematizadora (3b):

(3) a. H1: ¿Qué le parece a usted la Constitución?

H2: La cosa más bonita que tenemos, los españoles (ejemplo tomado de Hidalgo Downing 2003, 129).

b. $\quad$ ? -Pero el presidente de Brasil no puede fabricar por sí mismo medicinas contra el sida.

-Claro que sí: puede comerciar fármacos libremente, con respecto a las patentes.

Con todo, si la topicalización implica la presentación de un universo de aplicabilidad del predicado que sigue, es difícil admitir la existencia de tales topicalizaciones a la derecha, que pueden ubicarse mejor dentro de la idea de apéndice. ${ }^{5}$

La vinculación semántica entre el constituyente tematizado y la predicación principal puede ser variada. Así, en un ejemplo como:

(4) Respecto a ti, los coches no se conducen solos,

la existencia de topicalización garantiza la pertinencia discursiva del enunciado de la principal; el enunciado principal está intencionalmente dirigido al hablante al que se señala en la referencia metadiscursiva que se introduce con en cuanto $a$ como expresión de respectividad. Avala, pues, que un aserto como los coches no se conducen solos no es desafortunado contextualmente (por ejemplo, en un contexto conversacional en que se quiere recriminar a alguien su impericia o temeridad al volante).

5 Más bien estaríamos ante expresiones (de)limitativas, con independencia de que puedan usarse las mismas marcas para topicalizar y para delimitar-restringir (y aun así, unas están más especializadas que otras: en (12) será integrable en cuanto que y, como hablante, me resulta poco admisible con respecto $a$. 


\subsection{Los topicalizadores en su descripción sincrónica: presencia en lo hablado, elenco, denominación}

A grandes rasgos, se puede afirmar que las expresiones topicalizadoras son en español actual poco frecuentes en enunciados medialmente orales y concepcionalmente hablados, como pone de manifiesto Hidalgo Downing (2003) quien, a partir del análisis de distintos corpus orales, habla de sobrevaloración de esta clase de expresiones como introductoras de constituyentes topicalizados. De hecho, falta la mención a estos elementos en no pocos de los trabajos sobre organización discursiva, marcadores y cualquier otro asunto sobre conformación del texto desde una perspectiva enunciativa. En efecto, pese a la muy abundante producción teórica sobre partículas discursivas y mecanismos de conexión, en un principio las expresiones topicalizadoras apenas fueron atendidas en su funcionamiento sincrónico, propiedades y rasgos de uso. No se abordaron en trabajos sobre cohesión clásicos para el español tales como la conocida obra de Mederos (1988). Tampoco las trata explícitamente en su libro sobre conectores Montolío (2001, 155), aunque califica como organizador de la información a en lo que respecta al aparecer como encabezador de frase en uno de los textos comentados al hilo del conector de continuidad $a$ su vez. ${ }^{6}$

En la caracterización sincrónica de estos elementos se advierte una muy notable diversidad terminológica. En español, se las ha denominado «expresiones tematizadoras» (Uritani 1984; Hernanz/Brucart 1987, 82), «marcadores de tematización» (Perona 2000, 450); «expresiones topicalizadoras» (Contreras 1978, 98; Kovacci 1999, 754, n. 80), «marcadores de topicalización» para Gutiérrez Ordóñez (1997, 52) y Garcés Gómez (2002) o «marcadores discursivos de topicalización» (Cifuentes Honrubia 2001), entre otros nombres.

Por otra parte, cuando se habla de topicalizadores expresos se hace alusión a un repertorio de estructuras igualmente muy variado. Contreras $(1978,98)$ menciona como «expresiones topicalizadoras» a estructuras tan disímiles como en cuanto a y hablando de; Uritani (1984) da un elenco más nutrido: con respecto $a$, en relación con, en lo concerniente $a$, acerca de, por lo que atañe a, en lo tocante $a$, en / por lo que hace a, en lo pertinente a, a propósito de, en lo relativo a... Casado Velarde $(1998,66)$ agrupa dentro de la función textual «topicalización» o «tematización» a en cuanto $a$, por lo que se refiere $a$, por lo que respecta $a$, en lo

6 Sí parece darse más atención al funcionamiento de estas expresiones topicalizadoras en otros idiomas: tratan tangencialmente el funcionamiento de concerning o as for en inglés Keenan/ Schiefflin (1976) y Creider (1979), Fløttum (2000) se ocupa de quant à en francés y Caron/ Mohamadou (2000) describen algunos marcadores de topicalización propios de lenguas no indoeuropeas. 
concerniente $a$, en lo tocante a y a propósito de. Por su parte, Llorente Arcocha (1996, cap. 6) separa una serie de oraciones o cláusulas de tópico que sirven para presentar el tópico global con formas como es sobre, es referente a, yo era para.

\section{Respecto en el español de hoy: usos formales y valores}

En el español actual, respecto es integrante de locuciones con un significado común de limitación del ámbito al que afecta una parte del predicado en que se integra.

\subsection{Variaciones formales}

A diferencia de otro topicalizador como en cuanto $a$, que, tras una etapa de cierta variación formal (cuanto a, cuanto en) se fija en los Siglos de Oro con la estructura que hoy empleamos en español, respecto figura, hoy como ayer, en un repertorio de locuciones diversas. Separamos un total de trece expresiones, disociadas entre sí por las diferentes preposiciones que las rodean, sus distintas capacidades de integración en la frase e implicaciones significativas:

(5) a. Respecto a Sofía Loren, me parece una actriz excepcional.

b. No haré declaraciones respecto al nuevo proyecto.

c. Mostró su interés respecto al nuevo proyecto.

d. Respecto al año pasado, este año ha hecho mejor tiempo.

d'. Los precios de los inmuebles han bajado respecto al año pasado.

e. La posición de los planetas respecto al sol influye en su temperatura.

(6) a. Respecto de Sofía Loren, me parece una actriz excepcional.

b. No haré declaraciones respecto del nuevo proyecto.

c. Mostró su interés respecto del nuevo proyecto.

d. Respecto del año pasado, este año ha hecho mejor tiempo.

e. Los precios de los inmuebles han bajado respecto del año pasado.

f. La posición de los planetas respecto del sol influye en su temperatura.

(7) a. Al respecto de las opiniones del ministro, Luis declaró que no había crisis.

a'. Los precios de los inmuebles han bajado. Al respecto de lo cual, los consumidores muestran cierto escepticismo.

b. No haré declaraciones al respecto de las opiniones del ministro.

c. Mostró su interés al respecto del nuevo proyecto.

d. Al respecto del año pasado, este año ha hecho mejor tiempo. 
e. Los precios de los inmuebles han bajado al respecto del año pasado.

f. La posición de los planetas al respecto del sol influye en su temperatura. ${ }^{7}$

(8) a. El alcalde ayudará a las familias numerosas. Al respecto, les financiará los gastos escolares.

b. No haré declaraciones al respecto.

c. Mostró su interés al respecto.

(9) a. Con respecto a tu libro, será un verdadero éxito editorial.

b. No haré declaraciones con respecto al nuevo proyecto.

c. Mostró su interés con respecto al nuevo proyecto.

d. Los precios de los muebles han bajado con respecto al año pasado.

e. Con respecto al libro, la película gana en rapidez pero pierde en argumento.

f. La posición de los planetas con respecto al sol influye en su temperatura.

(10) a. Con respecto de tu libro, será un verdadero éxito editorial.

b. No haré declaraciones con respecto del nuevo proyecto.

c. Mostró su interés con respecto del nuevo proyecto.

d. Los precios de los inmuebles han bajado con respecto del año pasado.

e. La posición de los planetas con respecto del sol influye en su temperatura.

(11) a. El alcalde apostará por ayudar a las familias numerosas. A es(t)e respecto, éstas tendrán financiación de los gastos escolares.

b. No haré declaraciones a es(t)e respecto.

c. Mostró su interés a este respecto.

d. No contaban con fondos, por lo que organizaron a es(t)e respecto una asamblea extraordinaria.

(12) La inexistencia de fondos, a cuyo respecto organizaron una asamblea, se explica por la gestión ministerial.

(13) a. Por lo que respecta a tu libro, será un verdadero éxito editorial.

b. Nada confesó por lo que respecta al posible pago de comisiones.

c. Mostró su interés por lo que respecta al nuevo proyecto.

d. Los precios de los inmuebles han bajado por lo que respecta al año pasado. ${ }^{8}$

e. La posición de los planetas por / en lo que respecta al sol.

7 Se ha de constatar que los enunciados c, d, e, f de esta sección resultan poco admisibles para muchos de los informantes consultados. Otros muestran dudas por todas las frases de esta sección y prefieren en todos los casos un al respecto sin complementación o el paso a con respecto $a \mathrm{o}$ similares.

8 Para esta frase (y también para su paralela en el grupo que sigue) es preciso apuntar la existencia de cierta ambigüedad interpretativa. Una parte de los informantes consultados entiende por lo que respecta como comparativo (= 'los pisos han bajado este año frente a lo que respecta al año pasado'), otros como respectual (= 'los pisos bajaron el año pasado'), y otros como restrictivo (= 'si hablamos solo del año pasado'). 
f. Es provechoso por lo que respecta a capacidad de afinación.

f'. El balance ha sido negativo por lo que respecta a la participación.

f'. Es agudo por lo que a capacidad de percepción respecta.

(14) a. En lo que respecta a tu libro, será un verdadero éxito editorial.

b. Nada confesó en lo que respecta al posible pago de comisiones.

c. Mostró su interés en lo que respecta al nuevo proyecto.

d. $\quad$ ? Los precios de los inmuebles han bajado en lo que respecta al año pasado.

e. Es provechoso por lo que respecta a capacidad de afinación.

e'. El balance ha sido negativo por lo que respecta a la participación.

e”. Es agudo por lo que a capacidad de percepción respecta.

(15) Es más ventajoso, a todos los respectos, que vendas tu parte.

(16) Es más ventajoso, en todos los respectos, que vendas tu parte.

(17) Una casa es, en cierto respecto, una pequeña empresa.

Formalmente, las variaciones giran en torno a i) el valor nominal o verbal de la forma RESPECT*; ii) la preposición o preposiciones de las que se puede acompañar.

Tabla 1: Variación formal sincrónica en las configuraciones con respecto -ar

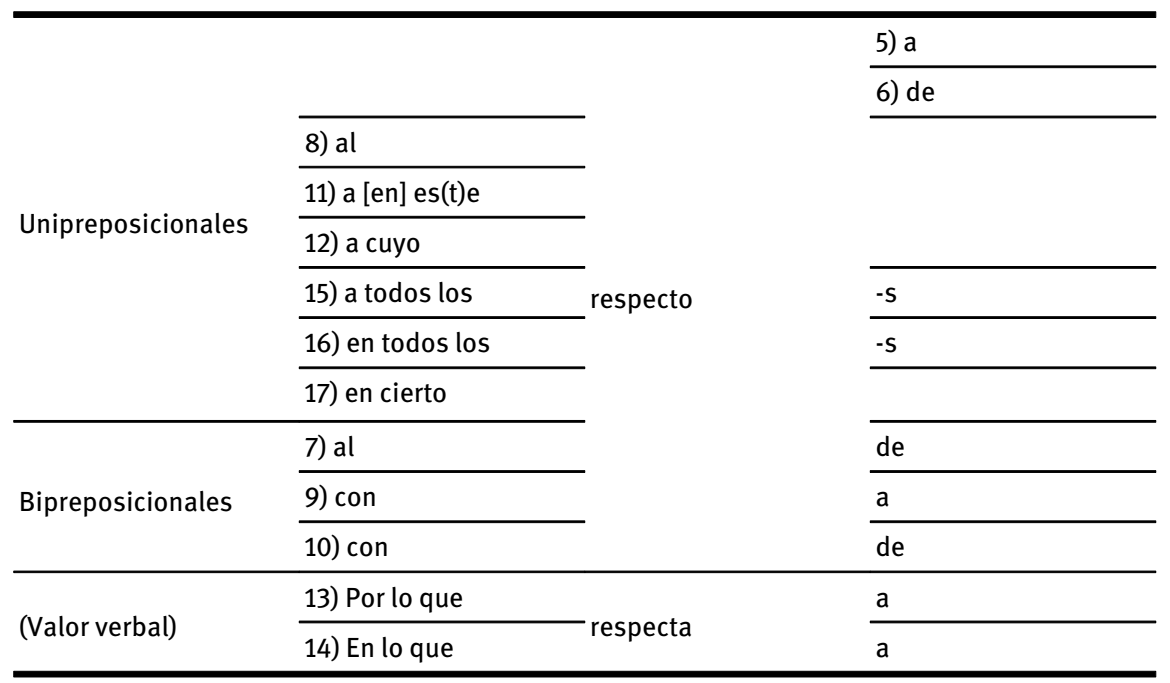

Dejo fuera de esta caracterización sincrónica a formas de muy inusitado reflejo textual como en respecto $a$, por respecto a que sí aparecen, en cambio, en la descripción diacrónica (Pons Rodríguez 2017). Más frecuentes son las variantes 
con $a$ de las estructuras con demostrativo y respecto: en es $(t)$ e respecto. ${ }^{9}$ También raras son las locuciones en que se implica, en lugar de respecto, la forma respective, de cuyos orígenes y vigencia actual me ocupé en Pons Rodríguez (2010).

\subsection{Valores de empleo}

Se pueden indicar algunas propiedades y significados comunes en tan amplio muestrario de formas, ${ }^{10}$ separables en dos grandes conjuntos. En primer lugar, las estructuras numeradas de 5 a 14, que conforman el centro de interés de este trabajo. En segundo lugar, las estructuras 15, 16 y 17 (en cierto respecto, a / en todos los respectos) en las que respecto es sustituible por sentido y que, como se observa, presentan menor capacidad de variación en cuanto a colocación, significado y propiedades.

En las estructuras 5 a 14 podemos deslindar dos grandes valores. El primero es el valor topicalizador, que se observa en los enunciados marcados con $a$. En todos los casos señalados, respecto se instala en un ámbito periférico a la oración (antepuesto en todos los ejemplos transcritos), introduciendo un complemento parafraseable respectualmente (hablando del año pasado), e intercambiable, por tanto, con otro topicalizador prototípico del español, en cuanto $a .^{11}$

Cabe mencionar el particular funcionamiento topicalizador de las estructuras con respecto en casos como (8.a: al respecto) y (11a: a es(t)e respecto), que a continuación ejemplifico a partir de un par de citas procedentes de un texto periodístico actual:

(18) A partir de ahora, Unicaja realizará «la mejor recepción de los documentos para garantizar su mejor disponibilidad», dijo Medel [...]. Al respecto, la entidad ya ha ofrecido a la Consejería de Cultura la posibilidad de filmar los documentos y exponer-

\footnotetext{
9 Como en la forma principal con $a$, son más frecuentes los casos de en este respecto que de en ese respecto; en el CREA la primera forma ofrece 55 casos en 34 documentos, en tanto que la segunda forma deja 6 casos en 6 documentos. Rarísimo es el hallazgo de formas en plural como a estos respectos o los respectos.

10 En Santos Río (2003) puede encontrarse una caracterización bastante completa de estas estructuras; con todo, he preferido reagrupar algunas de las formas e introducir caracterizaciones propias, por lo que no renuncio a detenerme en la caracterización sincrónica de estas expresiones.

11 Sería, pues, lo que Santos Río (2003) llamaba un circunstancial respectual disjunto. No son siempre equivalentes respectualidad disjunta y topicalización, por ello, haré uso de $r$. adjunta y de topicalización, y solo circunstancialmente de $r$. disjunta.
} 
los en la biblioteca pública Infanta Elena de Sevilla, gestionada por la Junta [...] A este respecto, el máximo responsable de Unicaja explicó que todas las razones para la adquisición de los documentos «giran en torno a nuestra condición de caja de ahorros» (El Mundo edición Andalucía, 22-11-2003, 51).

En ambos casos, se practica una topicalización, con la particularidad de que no se recupera explícitamente el constituyente topicalizado (y ello ocurre justamente con las únicas estructuras topicalizadoras unipreposicionales a la izquierda); establecen, pues, una referencia fórica a lo previo de límites más imprecisos que cualquier otro topicalizador, y esa imprecisión está, evidentemente, más acentuada en al respecto que en la variante con demostrativo, dada la funcionalidad prototípicamente señaladora de este elemento. Así, en el ejemplo transcrito, al respecto puede estar recuperando tanto el aserto anterior, como los tres enunciados anteriores, dado que conjuntamente también pueden brindar el marco de pertinencia discursiva en el que es oportuno y cobra toda su congruencia el anuncio de la posibilidad de filmar los documentos.

En comparación con el resto de las estructuras topicalizadas en que se integra respecto, tienen al respecto y a es(t)e respecto menor capacidad de ruptura de línea informativa y de apertura de una nueva. La diferencia es mayor si las comparamos con las estructuras 5, 6, 9, 13 y 14, que suelen topicalizar uno de los varios temas de una serie previa, como se observa en la cita que sigue, donde cada uno de los temas que se manejan (recesión e inflación) es retomado por un topicalizador con respecto:

(19) La clave de la reacción positiva de los mercados, sin embargo, debe buscarse en el alejamiento de los temores de una posible recesión, así como los que suscita una cierta reactivación de la inflación. Por lo que respecta al primero de ellos, deben tenerse en cuenta -como complemento a la buena cifra de evolución del paro- los indicadores conocidos a lo largo de la semana: un aumento mayor de lo previsto en el índice de confianza de los consumidores de agosto, un crecimiento de las rentas individuales en julio y un incremento de las ventas de viviendas nuevas en junio. Con respecto a la inflación, los mercados valoraron positivamente las bajas medias registradas en los salarios (1995, La Vanguardia, CREA).

Es decir, cada una de las aperturas con estos topicalizadores supondrá el cierre del tema previo, y ello no parece posible ni con los respectos que se acompañan de demostrativo ni con al respecto a secas, que no precisan ser precedidos de una serie temática y que, por tanto, no abren un eslabón en la cadena informativa, sino que apuntalan algo sobre lo previo. Esa incapacidad de rescatar expresamente lo topicalizado, las convierte, además, en expresiones con nula capacidad focalizadora, y por eso es difícil que se aparten del marco narrativo principal. Colaboran, como el resto de las configuraciones, en la progresión temática, pero parecen estar más al servicio de la conexión que de la progresión informativa; 
obsérvese, en este sentido, su posible equivalencia a conectores causales o meramente ilativos:

(20) a. El alcalde ayudará a las familias numerosas. \{Al respecto / Por eso / Y\}, les financiará los gastos escolares.

(21) a. El alcalde apostará por ayudar a las familias numerosas. \{A es(t)e respecto / Por eso / Y\}, éstas tendrán financiación de los gastos escolares.

Precisamente por esa incapacidad focal, introducen con especial frecuencia actos ilocutivos indirectos.

El segundo gran valor que atribuiremos a respecto es el de delimitador de pertinencia, y en este se recogen distintas especificaciones internas de transición continua: delimitador de pertinencia discursiva, no discursiva y paramétrica (comparativa o no comparativa). Será un delimitador de pertinencia discursiva (o adjunto de respectualidad) si posee complemento (en todos los casos marcados como b) que introduce el asunto o el tema sobre el que versa el predicado de la principal, del que no está separado mediante pausa. Con esta función, respecto estará ligado a verbos de decir o a predicados nominales que remiten a un acto de habla. Precisa, por tanto, de elementos con un referente que implique una producción discursiva, sobre la que el complemento con respecto puntualizará el asunto del que ese hecho versó.

Tres de las formas señaladas con esta función precisan de información complementaria, dada su limitada extensión de uso. En primer lugar, la forma con respecto de (10b), que puede resultar poco común para el hablante medio peninsular, tiene uso más frecuente como topicalizador que como delimitador. En segundo lugar, las estructuras (13) y (14) permiten un cambio de posición del complemento que introduce la circunstancia topicalizada: en/por lo que al pago de comisiones respecta, estructura aún más marcada y elaborada que las que presentan el verbo al inicio de la construcción. Por otra parte, son casos limítrofes aquellos en que una expresión encabezada por respecto puede estar funcionando como topicalizador o también como adjunto a un verbo; ocurre esta doble posibilidad especialmente en las muestras en que el predicado de la principal tiene como núcleo un verbo de implicaciones metadiscursivas:

(22) Decir que la angustia es señal de peligro es una expresión de valor limitado. Con respecto al dolor, también se emplea una expresión parecida. El dolor es señal de peligro (1966, Juan José López Ibor, Las neurosis, CORDE).

Hay también delimitación de pertinencia no discursiva cuando respecto fija el ámbito en el que la expresión a la que se adjunta tiene sentido. Ese establecimiento de un contorno de congruencia es común con el grupo anterior, pero, a 
diferencia de aquél, aquí no se presenta pertinencia en cuanto a dictum sino en cuanto a actum. A este grupo se adscriben ejemplos como marcados previamente como (c) y también (20)

Otro tipo de delimitación es la comparativa, en la que respecto introduce el valor de referencia del que nos servimos para establecer la situación en que se halla una determinada entidad. Respecto introduce una base de relación, un parámetro a partir del cual se mide el diferencial de una distancia (en su sentido no estrictamente físico): a distancia entre dos hitos temporales en la progresión de una propiedad, la distancia de una entidad en relación con otra, el diferente grado de dos entidades en su posesión de una propiedad. Distinguimos dos grupos:

- En el primero, esa fundamentación paramétrica se emplea para establecer una comparación (los ejemplos marcados como d). En estos enunciados observamos que respecto funciona como complemento adjunto del verbo de la frase (aunque pueda situarse antepuesto), introduciendo un parámetro que se pone en juego para cotejar el cambio que una determinada realidad enunciada en la frase ha sufrido. El verbo en todos los casos implicará algún tipo de cambio de estado (bajar, mejorar, empeorar, crecer, madurar) en una magnitud; se trata de acciones durativas, que señalan eventos que «se extienden a lo largo de un intervalo o periodo» (De Miguel 1999, 3030) o eventos de acabamiento gradual (como acercarse). Hay, pues, para los ejemplos citados supra, un lapso de tiempo que interesa fragmentar: respecto introduce el punto de referencia a partir del cual se considera el hecho del verbo, cuyo límite se colige de la temporalidad del verbo. La mejora, el crecimiento, el empeoramiento o el cambio se medirá desde el año pasado hasta el antepresente del enunciado. El punto de referencia no tiene por qué implicar la experimentación de un progreso en la acción:

(23) El precio de los pisos se ha estabilizado con respecto al año pasado.

Por el valor de 'fijación de un punto de referencia a partir del cual evaluar algo' que hemos adjudicado a respecto en las frases comentadas, se entiende que este complemento no pueda coaparecer con verbos que, por su aspecto delimitado, ya incluyen en sí un límite,

(24) ^Luisito se ha mudado de casa con respecto al año pasado.

Si bien los ejemplos expuestos han orientado nuestra descripción hacia el ámbito de la comparación entre dos momentos de una misma acción, hay que señalar que también podrá aparecer este valor ligado a una señalación de cambio de estado (los estados son aspectualmente durativos) y también es 
posible este esquema con un resultado, con un estado adquirido (poseído) (a, b) o con un mero estado:

(25) Luisito ha ganado peso con respecto al año pasado.

(26) a. Luisito ha suspendido muchas asignaturas respecto del año pasado.

b. El Betis tiene ahora diez puntos menos respecto del año pasado.

(27) Se sienten discriminados respecto a los peninsulares [ejemplo tomado de Santos Río 2003, 570 s.v. respecto $a, 2$ ].

- En segundo lugar, separamos aquellos casos en que respecto fundamenta un parámetro de referencia con implicación no comparativa. Se establece una base de relación entre dos entidades (por ejemplo, en los ejemplos marcados como f así como en 5e y 10e, a partir de la unión a un sustantivo con valor léxico que puede ligar mutuamente a dos realidades):

Existe también la delimitación aspectual o categorial. En estos casos respecto acota el espacio de acción de un término mediante la recuperación dentro de su extensión ontológica del aspecto que resulta pertinente para la enunciación que sigue

(28) Es una beca en lo que respecta a derechos, es un contrato con respecto a las obligaciones.

(14) e. Es provechoso por lo que respecta a capacidad de afinación.

e'. El balance ha sido negativo por lo que respecta a la participación.

e”. Es agudo por lo que a capacidad de percepción respecta.

\subsection{Estructuras en las que se integra respecto. Variación y frecuencia relativa}

La variación de frecuencia entre las diversas formas presentadas es muy diversa en el español actual. Algunos índices nos permiten medir la frecuencia relativa de cada una de ellas.

\subsubsection{Datos de corpus}

En la comprobación de la vigencia actual de ese elenco de formas señalado, y, también, a fin de sondear otras características no observadas en esta primera aproximación, nos ha parecido oportuno realizar una exploración sincrónica por 
la frecuencia de cada una de esas locuciones en las que respecto entra a formar parte. Para ello, hemos analizado los datos del corpus CREA de la RAE ${ }^{12}$ tomando un arco temporal convencionalmente restringido a 1996. En el cuadro que sigue reseñamos algunas variables de interés para esta primera aproximación estadística a la forma: frecuencia total, frecuencia documental y frecuencia geográfica relativa. $^{13}$

Tabla 2: Frecuencia total, documental y geográfica de las configuraciones con respecto

\begin{tabular}{lcrl}
\hline Locución & No de casos & No de documentos & Frecuencia geográfica \\
\hline a ese respecto & 9 & 9 & $\begin{array}{l}28,57 \% \text { España } \\
28,57 \% \text { México }\end{array}$ \\
\hline a este respecto & 143 & 101 & $\begin{array}{l}64,6 \% \text { España } \\
13,38 \% \text { México }\end{array}$ \\
\hline al respecto de & 17 & 17 & $\begin{array}{l}31 \% \text { España } \\
30 \% \text { Colombia }\end{array}$ \\
\hline con respecto a & 276 & $\begin{array}{l}43,2 \% \text { España } \\
9 \% \text { México }\end{array}$ \\
& 405 & $9,3 \%$ Venezuela \\
\hline con respecto de & & 48 & $90 \%$ México \\
\hline en lo que respecta & 56 & $42,2 \%$ España \\
& 125 & $20,6 \%$ México \\
\hline por lo que & & 48 & $75,6 \%$ España \\
respecta & 79 & 100 & $41,34 \%$ España \\
\hline Respecto a & 105 & $23,07 \%$ México \\
\hline
\end{tabular}

En este primer escrutinio de datos se advierten dos hechos de interés:

i) Las diversas expresiones en que se articula la forma respecto (o el verbo respectar) no son homogéneas en cuanto a preferencia numérica. En términos

12 La fuente fundamental de ejemplos para este trabajo está, de hecho, en la base de datos CREA de la RAE.

13 Por la falta de lematización de este corpus se consideran variables como la mayúscula o la aparición o no de contracciones al, del en el caso de locuciones que integran alguna de esas preposiciones. Las cifras estadísticas que presento implican, pues, la suma de los resultados aportados por cada una de esas variables una vez extraídas y unidas sus medias de empleo; se reseñan los índices de frecuencia más importantes. Exceptúo el caso de respecto $a$, que, por su posible aparición independiente o integrado en locuciones preposicionales, se ha explorado solo en posiciones de encabezamiento con mayúscula Respecto $a$. 
relativos, copan casi la mitad $(43,1 \%)$ de las ocurrencias de respect ${ }^{\star}$ en la cala temporal estudiada los casos de con respecto $a$; las particulares restricciones con que se ha estudiado la forma respecto al invitan a pensar que esta forma puede ser, al menos, tan frecuente como en lo que respecta. Siguen en frecuencia las formas con señalación fórica a es(t)e respecto. Se presentan como formas desfavorecidas numéricamente con respecto de y al respecto de, en tanto que no se encuentra documentación (o en número despreciable, inferior a 5 casos) para formas en las que respecto se une a relativo posesivo (en/a cuyo respecto), a demostrativo de tercer campo (a aquel respecto), a demostrativos en plural (es( $t$ ) os respectos), a cuantificador universal todo o al determinante no cuantitativo cierto. Igualmente no se dan locuciones como en el respecto del ni tampoco el rarísimo respective.

ii) Del conjunto de formas estudiado, la locución con respecto de es la única que muestra un perfil de uso diatópicamente marcado, con mayoritaria documentación en español americano (mexicano). Para el resto de casos, no se pueden colegir preferencias de uso en ese sentido, siendo que la frecuencia de ejemplos españoles (por encima de americanos en todos los casos salvo el mencionado con respecto de) puede explicarse por la propia constitución de fuentes del corpus manejado.

\subsubsection{Fuentes metalingüísticas}

Los diccionarios no especializados del español actual recogen la unidad respecto en locuciones con significados muy diversos. Tomando una muestra de doce diccionarios monolingües, ${ }^{14}$ muestra reducida pero representativa, encontramos ciertas preferencias en cuanto a la integración macroestructural de algunas unidades. Obviamente, debe ponderarse la trascendencia de esta información para la caracterización de las formas objeto de estudio. En este caso, que algunas locuciones sean privilegiadas en cuanto a su figuración macroestructural y que otras apenas estén presentes puede ser solo en parte reflejo de su mayor o menor frecuencia de uso (por tanto, de la mayor o menor consciencia de los lexicógrafos acerca de ellas). No puede descartarse la acción de la propia herencia de caudal léxico que se practica de diccionario en diccionario, rasgo caracterizador de buena parte de nuestra lexicografía. Reflejo los resultados en la tabla:

14 Se exponen con la clave que se les asigna en la bibliografía primaria que cierra este trabajo. 
Tabla 3: Presencia lexicográfica de las estructuras con respecto -ar en diccionarios actuales del español

\begin{tabular}{|c|c|c|c|c|c|c|c|c|c|c|c|c|}
\hline $\begin{array}{l}\text { DICCIONARIO } \\
\text { LOCUCIÓN }\end{array}$ & DUE & DES & DDE & DPL & DUEA & DEA & GDUEA & DAE & DRAE21 & DPD & LEMA & SALA \\
\hline Respecto de & * & & * & & * & * & & * & * & * & * & * \\
\hline Respecto a & * & & * & & * & * & & * & * & * & * & * \\
\hline Al respecto & * & * & * & * & * & * & * & * & * & * & * & * \\
\hline Al respecto de & & & & & & & * & & & * & & \\
\hline $\begin{array}{l}A \text { es }(t) e \\
\text { respecto }\end{array}$ & & & & & & * & & & & * & & \\
\hline $\begin{array}{l}\text { Con respecto } \\
a\end{array}$ & * & * & * & * & * & * & * & * & * & * & * & * \\
\hline $\begin{array}{l}\text { Con respecto } \\
\text { de }\end{array}$ & & * & * & * & & * & * & & * & * & & * \\
\hline $\begin{array}{l}\text { En lo que } \\
\text { respecta a }\end{array}$ & * & * & & * & & * & & & & & & * \\
\hline $\begin{array}{l}\text { Por lo que } \\
\text { respecta a }\end{array}$ & $\star$ & * & & * & & * & & & & & & \\
\hline Al respective & & & & & & * & * & * & & & * & \\
\hline Respective a & * & & & & & * & * & & & & & \\
\hline Respective de & & & & & & * & & & & & & \\
\hline $\begin{array}{l}\text { En lo } \\
\text { respective a }\end{array}$ & & & & & & * & & * & & & * & \\
\hline
\end{tabular}

Se observa cómo hay posibilidades combinatorias que no se recogen en ninguna de las fuentes consultadas: en todos los respectos, en ese respecto, otras que apenas tienen presencia ( $a$ este respecto, al respecto de) y otros esquemas que se repiten constantemente (al respecto, sin complemento, y con respecto a están en todas las fuentes consultadas). El Diccionario de Seco (DEA) es el que recoge el panorama más amplio de combinaciones.

En cuanto a la microestructura, son destacables algunas indicaciones dentro de las definiciones que pueden resultar de interés, como las siguientes:

- Caracterización de respecto: se suele definir con términos que implican 'relación', 'proporción', 'referencia' o con equivalencia a topicalizadores como en lo tocante.

- Relación con respectar: solo en algunos de los diccionarios se recoge como lema independiente el verbo respectar, en la mayoría remitiendo directamen- 
te a las expresiones en que puede figurar este verbo conjugado y en algunos se apunta algún dato más, como la naturaleza defectiva de la forma, solo usado en las terceras personas del singular (DAE, DUEA), su carácter intransitivo (GDUEA) o impersonal (SALA).

- Caracterización prosódica: en DEA se califica como átona la forma respecto «especialmente si no va precedida de con» (s.v.). Cabe señalar (aunque se trate de un texto que queda fuera de este primer ámbito de interés lexicográfico) que solo en este punto de la tonicidad o atonicidad de respecto incluye el Esbozo académico (1973, 1.5.4.a.7) información acerca de esta voz (en la forma respecto $a$ ) a la que incluye bajo el marbete de «preposiciones simples y compuestas» y junto con una lista de elementos preposicionales donde, además de los ortodoxos, están también conforme $a$, mediante, frente a o junto. Según la RAE, todo ese conjunto de formas pertenece al grupo de palabras inacentuadas, aunque en nota aclara que respecto es "palabra acentuada y de otra categoría cuando se halla en otros contextos».

- Calificaciones normativas: DDE y DUEA califican como incorrecta con respecto de, si bien la Academia, en su Panhispánico de dudas da por válidas todas las formas que recoge, incluida con respecto de. María Moliner calificaba de popular el giro respective $a$.

En Pons Rodríguez (2005) me ocupé de los rasgos y características con que estos elementos figuran en un grupo textual muy determinado: los manuales de español como lengua extranjera, en los que, sobre todo en niveles intermedios y superiores, se introduce, de forma diversa, la enseñanza de expresiones topicalizadoras. Sin duda, el más presente en manuales de ELE era en cuanto y, tras él, las expresiones configuradas a través del elemento respecto, por orden: respecto a, respecto de, a este respecto, al respecto, con respecto a, en / por lo que respecta a. Como se observa, hay algunas variaciones con la frecuencia que presentan algunas de estas locuciones en cuanto a plasmación lexicográfica.

\section{Descripción categorial}

Estamos ante un conjunto de formas muy distintas, en unas figura respecto como elemento invariable, en otros casos permite estar acompañado de demostrativos, indefinidos etc., por lo que parece que nos encontremos ante un sustantivo, en otras es un verbo conjugado que permite alternancias con otros como referirse o tocar con un sema común de posibilidad de señalar a un elemento afectado por la aserción siguiente. Todas estas variaciones son una herencia del proceso de gramaticalización por el que ha atravesado respecto (Pons Rodríguez 2017) desde 
una estructura limitativa calcada del neolatín a su salto tardío hacia la topicalización en el español del siglo XIX.

En efecto, bajo este nada corto repertorio formal del español actual parecen latir grados de gramaticalización muy diversos, desde formas muy gramaticalizadas como al respecto a formas menos gramaticalizadas como en lo que respecta (donde el verbo permite las alternancias señaladas) o a todos los respectos, donde el valor léxico de 'consideración, punto de vista' de respecto se conserva, como manifiesta la posibilidad de reemplazarlo por aspecto o punto de vista:

$+$

al respecto por/en lo que respecta a en todos los respectos

Esta diversidad interna, venida de la distinta situación en que se encuentra cada forma desde un punto de partida que está en el sustantivo respecto tomado desde el latín, explica las dificultades para caracterizar categorialmente a este conjunto de formas en sincronía.

Sobre la caracterización y etiquetado de estas formas, el interrogante está en la posibilidad de considerar marcadores discursivos a estas expresiones topicalizadoras, cuestión de la que se ocupó de forma pionera Cifuentes Honrubia (2003), quien afirmaba:

«debemos aceptar la pertinencia de unos marcadores discursivos de topicalización o tematización, utilizados para resolver una de las tareas fundamentales de la organización de la acción discursiva: la presentación de la entidad tópica [...] hay determinadas unidades complejas que tienen un alto grado de gramaticalización, y que pueden ser entendidas como unidades fraseológicas. Estas unidades fraseológicas pueden desarrollar también la función de marcador discursivo de topicalización, y algunas de ellas han sido consideradas como locuciones prepositivas» (ib., 145, 147).

Cifuentes Honrubia examina las distintas propiedades gramaticales que la bibliografía sobre marcadores señala como propias de estas formas, y concluye, a partir de la revisión de tales características en formas como en cuanto a, en lo que concierne etc. que se puede hablar de un grupo de marcadores discursivos de topicalización. ${ }^{15} \mathrm{Tal}$ es también la postura de Garcés Gómez (2002), que advierte

15 Aborda también un asunto bastante más peliagudo, el del estatuto que tendrían algunos de estos elementos en relación con las locuciones prepositivas. Separa este autor marcadores discursivos, topicalizadores y locuciones prepositivas, que pueden coincidir en algunos casos. La clave parece estar, según este autor, en el grado de gramaticalización que presenta cada forma, aunque no queda claro el tipo de gradación que se da de una categoría a otra. «Hay marcadores 
no obstante del hecho de que «[1] os sintagmas precedidos por en cuanto $a$, por lo que se refiere $a$, por lo que respecta a... no siempre funcionan como tópicos externos a la oración, sino que pueden integrarse en la misma y cumplir una determinada función sintáctica». Granvik $(2014,78)$, por su parte, estudia los usos de acerca de, en torno a/de y con respecto a en español como locuciones prepositivas que, al igual que las preposiciones de y sobre, "han ido entrando en el paradigma de los marcadores de tema /asunto del español».

Además de estas propuestas, se ha indagado (Santos Río 2003) sobre la posibilidad de que estemos ante locuciones preposicionales. Consideremos dos factores. En cuanto al grado de fijación de las locuciones, una forma como al respecto puede variar en cuanto a posibilidad de adjunción del segmento [prep + $\mathrm{sN}$ ] (cf. 14-15), perdiéndose el valor fórico de la forma incrementada. En el caso considerado, frente a otros empleos señaladores de ámbito de limitación de enunciados esa posibilidad de aparecer sin complementos que especifiquen el elemento al que señalan singulariza a respecto: No tengo quejas al respecto // No tengo quejas $\left\{{ }^{*}\right.$ sobre / ${ }^{*}$ acerca\}. La variación que se produce entre respecto de / a en sincronía no debería ser tomada en cuenta en este sentido. Aunque, según Santos Río (2001, 33), «si la preposición que suele seguir al 'núcleo' del segmento (de en a base de) puede variarse, ello es, en principio, señal de baja o nula locucionalidad», este criterio no es aplicable en casos como en relación con, en relación a o respecto a, respecto de en los que «el significado del segmento completo no varía y el cambio preposicional afecta solo o bien a la preferencia idiolectal: las dos variantes suelen tener el mismo grado de locucionalidad».

En segundo lugar, en cuanto al grado de idiomaticidad, respecto es, para el hablante medio de español, una forma con valor léxico poco claro; en ese sentido, se puede hablar de pérdida de conciencia etimológica de núcleo (acompañada, además, de atonicidad) y de presencia de elemento léxico exclusivo (es decir, estas expresiones presentan un elemento léxico que no se usa fuera de ese grupo de formas). Pero, en cambio, en los casos en que la expresión contiene conjugado el verbo respectar, estamos ante formas menos gramaticalizadas, más alejadas del carácter de locución, por darse la posibilidad de inversión dejando el término preposicional inserto. Esto es, ante oraciones con expresiones donde respectar está implicado, topicalizadoras o meramente delimitadores de ámbito de pertinencia predicativo:

de topicalización que pueden ser considerados locuciones prepositivas, pero no por funcionar como marcadores con locuciones prepositivas, pues, aun estando gramaticalizados y encabezando un complemento, puede que no se comporten sintácticamente como preposiciones, y puede que, semánticamente, su significado léxico domine sobre el instrumental, así, por ejemplo, en lo que se refiere $a »$ (Cifuentes Honrubia 2003, 157). 
(29) a. Por lo que respecta a las elecciones, estaré de viaje y no votaré.

b. Por lo que a las elecciones respecta, estaré de viaje y no votaré.

(30) a. El precio ha bajado en lo que respecta a costes, pero no a impuestos.

b. El precio ha bajado en lo que a costes respecta, pero no a impuestos.

la posibilidad de la existencia de las variantes (b) indica que estamos ante una forma verbal con vida, por eso se puede invertir el verbo. Con todo, la posibilidad de (b) es menos natural con respectar que con otros verbos como referirse («en lo que a costes se refiere»). Por ello, la idea de locucionalidad parece más difícil para las expresiones con referir que con respectar.

\section{Conclusiones}

Por tratarse de un elemento dedicado a la organización global del discurso y situarse en la periferia oracional, la expresión descrita resulta de gran interés. En este trabajo nos hemos acercado al ámbito de la organización informativa del texto para analizar a respecto como estructurador de la información, observando el funcionamiento en español actual de esta unidad. Particularmente, la atención a la diversidad de estructuras en que se integra esta voz pone de manifiesto su amplia versatilidad funcional: desde la topicalización como operación en la periferia del enunciado a la limitación de pertinencia en el interior de estructuras integradas en el núcleo verbal.

\section{Bibliografía}

Beaudrie, Sara, Refinando la noción de foco en español: cuestiones semánticas y sintácticas, Arizona Working Papers in SLAT 12 (2005), 21-30.

Borreguero Zuloaga, Margarita, Cómo se organiza la información textual. Reflexiones sobre la didáctica de la escritura, Cuadernos de Filología Italiana 10 (2003), 27-48.

Borreguero Zuloaga, Margarita, La progresión temática textual: algunos problemas pendientes, in: Villayandre Llamazares, Mirka (ed.), Actas del V Congreso de Lingüística General, Madrid, Arco Libros, 2004, 445-458.

Caron, Bernard/Mohamadou, Aliou, La spécification du terme topique en haoussa et en peul. Vers une caractérisation contrastive de la thématisation et de la focalisation, in: Claude Guimier (ed.), La thématisation dans les langues, Frankfurt Main, Lang, 2000, 65-79.

Casado Velarde, Manuel, Lingüística del texto y marcadores del discurso, in: Martín Zorraquino, María Antonia/Montolío Durán, Estrella (edd.), Los marcadores del discurso. Teoría y análisis, Madrid, Arco Libros, 1998, 55-70.

Cifuentes Honrubia, José Luis, Marcadores discursivos, topicalizadores y locuciones prepositivas en español, Lingüística Española Actual 23:2 (2001), 237-255. 
Cifuentes Honrubia, José Luis, Locuciones prepositivas. Sobre la gramaticalización preposicional en español, Alicante, Publicaciones de la Universidad de Alicante, 2003.

Contreras, Heles, El orden de palabras en español, Madrid, Cátedra, 1978.

CORDE = Real Academia Española (ed.), Corpus Diacrónico del Español (CORDE), <http://corpus. rae.es/cordenet.html> [Fecha de consulta: noviembre 2015].

CREA = Real Academia Española (ed.), Corpus de referencia del español actual (CREA), <http:// corpus.rae.es/creanet.html> [Fecha de consulta: noviembre 2015].

DAE = Lucena Cayuela, Núria (ed.), Diccionario de uso del español de América y España, Barcelona, Vox, 2002.

DDE = De las Heras Fernández, Juan Antonio, Diccionario didáctico de español intermedio, Madrid, SM, 1994.

De Miguel, Elena, El aspecto léxico, in Bosque, Ignacio/Demonte, Violeta (edd.), Gramática descriptiva de la lengua española, Madrid, Espasa-Calpe, 1999, 2977-3060.

DEA = Seco, Manuel, et al. (edd.), Diccionario del español actual. Madrid, Aguilar, 1999.

DES = Diccionario esencial Santillana de la Lengua, Madrid, Santillana, 1991.

DPD = Real Academia Española, Diccionario panhispánico de dudas, Madrid, Real Academia Española et al., 2005.

DPL = Gallart Gual, Esther (ed.), Diccionario práctico. Locuciones, Barcelona, Larousse, 1995.

DUE = Moliner, María, Diccionario de uso del español, Madrid, Gredos, 1991.

DUEA = Maldonado González, Concepción (ed.), Clave. Diccionario de uso del español actual, Madrid, SM, 2008.

Dufter, Andreas/Octavio de Toledo, Álvaro, Left sentence Peripheries in Spanish. Diachronic, Variationist and Comparative Perspectives, Amsterdam, Benjamins, 2014.

Fernández Soriano, Olga, Sobre el orden de palabras en español, Dicenda: Cuadernos de Filología Hispánica 11 (1993), 113-152.

Ferrari, Angela/Borreguero Zuloaga, Margarita, La interfaz lengua-texto. Un modelo de estructura informativa, Traducción al español de Pura Guil, Madrid, Biblioteca Nueva, 2008.

Firbas, Jan, On defining the theme in functional sentence analysis, Travaux linguistiques de Prague 1 (1964), 267-280.

Fløttum, Kjersti, «Quant à»: thématisateur et focalisateur, en Guimier, Claude (ed.), La thématisation dans les langues. Actes du colloque de Caen, Bern, Lang, 2000, 135-149.

Garcés Gómez, María Pilar, Adverbios de topicalización y marcadores de topicalización, Romanistisches Jahrbuch 53 (2002), 355-382.

GDUEA = Sánchez, Aquilino (ed.), Gran diccionario de uso del español actual, Madrid, SM, 2001.

Granvik, Anton, Hablando «de», «sobre» y «acerca de» la gramaticalización y la lexicalización: Panorama diacrónico de las relaciones entre preposiciones y locuciones prepositivas dentro del campo semántico de tema/asunto, in: Girón Alconchel, José Luis/Sáez Rivera, Daniel (edd.), Procesos de gramaticalización en la historia del español, Madrid/Frankfurt am Main, Iberoamericana/Vervuert, 2014, 77-117.

Gutiérrez Ordóñez, Salvador, Temas, remas, focos, tópicos y comentarios, Madrid, Arco Libros, 1997.

Halliday, Michael, Notes on transitivity and theme, Journal of Linguistics 3 (1967), 199-244.

Hernanz, María Luisa/Brucart, José María, La sintaxis. 1. Principios teóricos. La oración simple, Barcelona, Crítica, 1987.

Hidalgo Downing, Raquel, La tematización en el español hablado, Madrid, Gredos, 2003. 
Keenan, Elinor/Schiefflin, Bambi, Foregrounding referents: a re-consideration of left-dislocation in discourse, in: Proceedings of the 2nd Annual Meeting of the Berkeley Linguistics Society, Berkeley, Berkeley University Press, 1976, 240-257.

König, Ekkehard, The meaning of focus particles. A comparative perspective, London, Routledge, 1991.

Kovacci, Ofelia, El adverbio, in: Bosque, Ignacio/Demonte, Violeta (edd.), Gramática descriptiva de la lengua española, Madrid, Espasa-Calpe, 1999, 705-786.

LEMA = Battaner Arias, Paz (ed.), Diccionario de la Lengua Española lema, Barcelona, Vox-Spes, 2001.

Llorente Arcocha, María Teresa, Organizadores de la conversación, Salamanca, Universidad Pontificia de Salamanca/Caja Salamanca y Soria, 1996.

Martín Butragueño, Pedro, Aspectos prosódicos de la tematización lingüística. Datos del español de México, El Colegio de México, coloquio de 23-27 octubre de 2006, <http://lef.colmex. $\mathrm{mx} /$ Sociolinguistica/Entonacion\%20del\%20espanol\%20mexicano/Aspectos\%20prosodicos\%20de\%20la\%20tematizacion.pdf> [fecha de consulta: 19-11-2017].

Mederos, Humberto, Procedimientos de cohesión en el español actual, Tenerife, Excmo. Cabildo Insular de Tenerife, 1988.

Mendieta, Eva/Medina, Isabel, Anteposición de objeto en el habla culta de México y Madrid, Revista Española de Lingüística 27 (1997), 447-477.

Montolío, Estrella, Conectores de la lengua escrita, Barcelona, Ariel, 2001.

Perona, José, La cohesión textual y los enlaces extraoracionales, in: Alvar, Manuel (edd.), Introducción a la lingüística española, Barcelona, Ariel, 2000, 445-462.

Pons Rodríguez, Lola, Los tematizadores en los manuales de ELE, in: Las gramáticas y los diccionarios en la enseñanza del español como segunda lengua: deseo y realidad. Actas del XV Congreso Internacional de ASELE, Sevilla, Universidad de Sevilla, 2005, 683-692.

Pons Rodríguez, Lola, La elaboración léxica desde modelos latinos. Tres estudios de caso en el castellano medieval (inclusive, exclusive, respective), in: Castillo, Mónica/López Izquierdo, Marta (edd.), Modelos latinos en la Castilla medieval, Madrid/Frankfurt am Main, Iberoamericana/Vervuert, 2010, 81-111.

Pons Rodríguez, Lola, Deudas latinas y variaciones romances en la creación del marcador de topicalización «respecto», Verba. Anuario Galego de Filoloxia 44 (2017), 133-167.

Real Academia Española, Esbozo de una nueva gramática de la lengua española, Madrid, Espasa-Calpe, 1973.

SAL = Gutiérrez Cuadrado, Juan (ed.), Diccionario Salamanca de la Lengua Española, Salamanca, Santillana, 1996.

Santos Río, Luis, Líneas generales del Diccionario del español de todos, Salamanca, Kadmos, 2001.

Santos Río, Luis, Diccionario de partículas, Salamanca, Luso-Española de Ediciones, 2003.

Silva-Corvalán, Carmen, Topicalización y pragmática en español, Revista Española de Lingüística 14 (1984), 1-20.

Uritani, Nozomu, Sobre el tematizador «en cuanto a», Español Actual 42 (1984), 5-21.

Weil, Henri, De l'ordre des mots dans les langues anciennes comparées aux langues modernes, Paris, Joubert, 1844.

Zubizarreta, María Luisa, Las funciones informativas: tema y foco, in: Bosque, Ignacio/Demonte, Violeta (edd.), Gramática descriptiva de la lengua española, Madrid, Espasa-Calpe, 1999, 4217-4244. 